\title{
Renal Graft Vesicoureteral Reflux Survival and Oucome in Pediatric Recipients; Single Centre Experience
}

\author{
Mohmamed H. Kasem*, Tarek KH..Fathelbab*, Fatina Fadel**, \\ Hani Abdel-Raouf Morsi*** and Ayman F. Hussein* \\ * Department of Urology, Minia University Hospitals, Minia, Egypt \\ ** Pediatric renal transplantation unit, Cairo children's hospital, Cairo University, Cairo, Egypt \\ ***Department of Pediatric Urology, Cairo children's hospital, Cairo University, Cairo, Egypt
}

\begin{abstract}
Objective: The goal of this study was to evaluate pediatric post transplant vesicoureteral reflux (VUR) in terms of graft's Urinary Tract Infections (UTIs) episodes, graft outcome and survival in comparison to grafts that don't have reflux. Subjects and Methods: A total of 145 pediatric patients who underwent kidney transplantation at our transplantation unit between 2008 and January 2019 and suited our inclusion criteria were included in this retrospective cohort study and a voiding cystourethrography (VCUG) was done to them and categorized into main groups; group (A) refluxing graft group ( $n=66)$ and group (B) non refluxing graft group $(n=79)$. Baseline donor and recipient demographic characteristics, post transplant serum creatinine level at different points, graft outcome, and UTIs were listed. Post transplantation (Tx) UTIs and graft outcome were compared between both groups. Glomerular Filtration Rate (GFR) was measured using Shwartz formula. Frequency- Volume Chart (FVC) and UroDynamic Study (UDS) were used to identify Lower urinary Tract (LUT) condition and helped us regarding decision making in management of graft VUR. Results: Mean \pm SD age at time of Tx was $8.7 \pm 3.4 \mathrm{y}$ in group A versus $8.9 \pm 2.8 \mathrm{y}$ in group $\mathrm{B}, \mathrm{P}$ value $=0.667$. Regarding sex, 46 patients $(69.7 \%)$ were male in group $\mathrm{A}$, while 56(70.9\%) were male in group $\mathrm{B}, \mathrm{p}$ value $=0.876$. UTI occurrence was similar between groups $\{40(60.6 \%)$ patients in group A versus 49 (62\%) patients in group B, P value $=0.861$ but UTIs episodes (more than 3 episode per year) was $20(30.3 \%)$ patients in group A versus $1(1.3 \%)$ patient, $\mathrm{P}$ value $=0.001$. Both groups were similar in terms of 5 -year (100\% versus $98.5 \%)$, and 10 -year (76.2\% versus $84.7 \%$ ) graft survival. Conclusions: There was no difference between refluxing and non refluxing graft in terms of UTIs occurrence, graft function and survival. Frequency of UTI episodes was more in the refluxing graft like any native kidney primary VUR.
\end{abstract}

Keywords: Renal transplantation; graft veisicoureteral reflux; graft VUR outcome, graft VUR treatment

\section{Introduction}

There is much debates in the literature regarding graft VUR and if it has harmful effect on renal graft survival or not. There is also debates about doing routine VCUG post Tx to diagnose reflux. Some authors recommend to do routine VCUG for diagnosing it and to do quick management believing from them about its dangerous affection while others don't recommend routine using based on their concept that graft VUR is not as same as primary VUR in terms of kidney affection and deterioration $^{[1]}$, so we conduct this study to know much about it and complete the shortage in the literature regarding it. This study aims to evaluate the effect of graft reflux and its effect on graft outcome and survival in pediatrics.
In fact, asymptomatic graft reflux is common but the true prevalence in pediatrics is still unknown since routine voiding VCUG after transplantation is not a routine work ${ }^{[2]}$. It is not a surprise that the diagnosis of reflux in pediatric recipients increases with the time that passed since TX and occurs regardless of surgical technique that was used ${ }^{[3]}$. Dimercaptosuccinic acid (DMSA) scan was done routinely in all recipients and VUR is common reaching from 34 to $70 \%{ }^{[2]}$. Patients with symptomatic VUR presented with recurrent attacks of UTIs as an initial clinical symptom and needs surgical management in the form of endoscopic injection as recommended by Akiki, et al., ${ }^{[4]}$.

Renal Graft Vesicoureteral Reflux Survival and Oucome in Pediatric Recipients; Single Centre Experience 


\section{Methods}

This study was a retrospective cohort study including 145 patients who less than 18 years old and received renal graft with suspicion to have VUR in the period between 2009 and March, 2019 including cases which were done during conducting the study and followed for at least 1 year post Tx at Cairo pediatric university hospital. Selection of patients who suited our inclusion criteria to be enrolled in the study after a consent was taken from the recipient's parents, data was collected from patient's files. Patient's inclusion criteria:

(1) Recipients who had 18 years old or less when they underwent renal Tx with suspicious of having graft VUR in the form of recurrent UTIs episodes either with normal LUT or abnormal LUT;

(2) Recipients with symptoms suggesting post

Tx lower urinary tract dysfunction (LUTD,

(3)Recipients with graft hydronephrosis after exclusion any obstructing cause;

(4) Recipients who had a renal graft with at least one year follow up post Tx with suspicious with suspicious of having graft VUR. We excluded who with the following criteria:

(1) Patients who refused to participate into the study; (2) Patients more than 18 years old;

(3) Patients with post Tx follow up less than one year.

All donors had renal isotope scan to determine the differential function of both kidneys to select which had less split function. All patients had pre Tx VCUG as a routine protocol to exclude native kidney VUR. During Tx, ureteral anastomosis was done by anti refluxing Lich-Gregoir technique in all sample into native bladder even in augmented bladder. Before doing VCUG, urine analysis was done, if there was pyuria, the procedure was delayed until infection had been resolved. An Iodinated contrast media was injected after cleaning the external genitalia with antiseptic solution before catheterization. Retrograde filling the bladder until capacity was reached according to age adjusted formula; \{age (years) +2 ) x 30$\}$ in infants more than 1year old and children. Multiple images were taken under fluoroscopy during filling, voiding and lastly post void film was obtained. The following were recorded; VUR occurrence at which phase?, bladder shape and capacity, posterior urethra during voiding and Post void residual (PVR) volume and. VUR was graded according to the International Reflux Study.

Patients were categorized into two main groups; group (A) comprised of 66 patients with post Tx VUR (Figure 1 - 2), and group (B) comprised of 79 patients who didn't have reflux. Also into 4 subgroups; (groupA1), refluxing graft with normal LUT ( $\mathrm{n}=32$ ), (group A2), refluxing graft with abnormal LUT ( $n=34)$, group (B1), non refluxing graft with normal LUT $(n=58)$ and lastly (group B2), non refluxing graft with abnormal LUT $(\mathrm{n}=21)$. Comparison between 2 main group based on serum creatinine, UTIs occurrence and its episodes. FVC was used to assess LUT function and its results were documented. UDS eval-uation was done to detect at what phase and at what pressure VUR occurred, $(n=106,100$ patients without anaesthesia and 6 patients only under sedation).

As we didn't have video UDS, we used combination protocol of VCUG plus conventional cystomery to detect at which phase and at which pressure the reflux was occurred. In group (A), the (Maximum cystometric capacity) MCC was calculated roughly by subtracting the PVR from the total infused volume in patients with normal anatomical and functional bladders, while in patients with anatomical or functional bladder abnormality, we can't subtract the PVR from the total infused volume as the PVR in this cases due to residuals in the bladder beside the reflux residual volume so the total infused volume was recorded as a MMC.

Short term and long term complications in both groups were documented. Long term graft function was measured by estimated Glomerular Filtration Rate (eGFR) using pediatric Schwartz, 2009 formula, also graft survival was assessed using the suitable statistical test. The effect of multi variables on graft outcome was also assessed.

\section{Statistical analysis}

Kolmogorov-Smirnov test were used to detect data distribution normality and we documented median (range) values. Mean \pm standard deviation (SD) was used for continuous variables, while frequencies and percentages in nominal data.

Comparison of parametric numerical variables for 2 groups was done using Student's t test,

Renal Graft Vesicoureteral Reflux Survival and Oucome in Pediatric Recipients; Single Centre Experience 
while ANOVA test and post Hoc test were used when comparing more than 2 groups.

Comparison between 2 categorical data, Chi square test $(\chi 2)$ was used. Comparison of non parametric numerical variables between the 2 groups was done using Mann-Whitney test, while Kruskal Wallis test was used when comparing more than 2 groups.

Assuming significance at values less than $(0.05)$, statistical analysis on renal graft survival and patient survival was done by using KaplanMaier curves and significance was done by log rank test, while linear regression analysis was done to study the impact of different confounders on GFR ( $\mathrm{ml} / \mathrm{min})$. All statistical calculations were done by using computer programs SPSS (statistical package for social science) (version 16).

\section{Results}

Baseline demographic characteristics in groups and subgroups

We had total sample $(n=145)$ with 66 patients in Group A and 79 patients in group B. Regarding sex, we had 46 male and 20 female patients in the group A, while 56 male and 23 female patients in group $\mathrm{B}, \mathrm{p}$ value $=0.876$, other recipients characteristics was presented in tables (1-2). Mean time passed since transplantation \pm SD in group A and B was $4.7 \pm 2.8 \mathrm{y}$ and $6.4 \pm 2.6 y$, respectively, $\mathrm{p}$ value $=0.001$. Mean time passed to diagnose VUR \pm SD was $2 \pm 1 \mathrm{y}$. Mean ischemia time $(\mathrm{y}) \pm \mathrm{SD}$ was $45.2 \pm$ $8.3 \mathrm{y}, 38.4 \pm 4.3 \mathrm{y}$ in group A and B respectively, $\mathrm{p}$ value $=.0 .001$. Post transplant LUTD occurred in $38(57.6 \%$ ) patients in group $\mathrm{A}$ and 32 $(40.5 \%)$ patients in group $\mathrm{B}, \mathrm{p}$ value $=0.041$. Treatment of bladder dysfunction and cause specific surgery (Table3).

Table (1): Recipient's demographic in both groups

\begin{tabular}{|c|c|c|c|}
\hline \multirow{2}{*}{ Variable } & Group A & Group B & \multirow[b]{2}{*}{$p$ value } \\
\hline & $\mathrm{N}=66$ & $\mathrm{~N}=79$ & \\
\hline $\begin{array}{l}\text { Recipient sex }(\%) \\
\text { Male } \\
\text { Female }\end{array}$ & $\begin{array}{c}46(69.7 \%) \\
20(30.3 \%)\end{array}$ & $\begin{array}{c}56(70.9 \%) \\
23(29.1 \%)\end{array}$ & 0.876 \\
\hline $\begin{array}{c}\text { Recipient age group at time of interview }(\mathbf{y}) \\
\text { Early childhood } \\
\text { Late childhood } \\
\text { Adolescence }\end{array}$ & $\begin{array}{c}6(9.1 \%) \\
23(34.8 \%) \\
37(56.1 \%)\end{array}$ & $\begin{array}{c}2(2.5 \%) \\
7(8.9 \%) \\
70(88.6 \%)\end{array}$ & $<0.001 *$ \\
\hline $\begin{array}{c}\text { Recipient age at time of transplantation }(\mathbf{y}) \\
\text { early childhood } \\
\text { late childhood } \\
\text { Adolescence }\end{array}$ & $\begin{array}{l}22(33.3 \%) \\
31(47.0 \%) \\
13(19.7 \%)\end{array}$ & $\begin{array}{c}15(19.0) \\
53(67.1 \%) \\
11(13.9 \%)\end{array}$ & $0.046^{*}$ \\
\hline
\end{tabular}

* Significant difference at $\mathrm{p}$ value $<0.05$; Chi Square test 
Table (2): Recipient's demographic data in both main group

\begin{tabular}{|c|c|c|c|}
\hline \multirow[t]{2}{*}{ Variable } & Group A & Group B & \multirow{2}{*}{$p$ value } \\
\hline & $(\mathrm{N}=66)$ & $(\mathrm{N}=79)$ & \\
\hline $\begin{array}{l}\text { Present recipient weight }(\mathbf{k g}) \\
\text { Mean } \pm \text { SD }\end{array}$ & $42.2 \pm 15.3$ & $49.1 \pm 13.9$ & $0.005^{*}$ \\
\hline $\begin{array}{l}\text { Recipient weight at time of } \\
\text { transplantation }(\mathrm{Kg}) \\
\text { Mean } \pm \text { SD }\end{array}$ & $28.6 \pm 9.8$ & $31.5 \pm 10.3$ & $0.049 *$ \\
\hline $\begin{array}{l}\text { Recipient age at time of } \\
\text { transplantation }(y) \\
\text { Mean } \pm \text { SD }\end{array}$ & $8.7 \pm 3.4$ & $8.9 \pm 2.8$ & 0.667 \\
\hline $\begin{array}{l}\text { Onset of dialysis ( } \mathbf{y}) \\
\text { Mean } \pm \text { SD }\end{array}$ & $6.9 \pm 2.8$ & $7.3 \pm 3$ & 0.545 \\
\hline $\begin{array}{l}\text { Duration of dialysis (y) } \\
\text { Median (Range) }\end{array}$ & $1(0.3-6)$ & $2(0.5-6)$ & 0.718 \\
\hline $\begin{array}{l}\text { Pre-emptive transplantation } \\
\text { Yes }\end{array}$ & $17(25.8 \%)$ & $35(44.3 \%)$ & $0.020 *$ \\
\hline
\end{tabular}

* Significant difference at $\mathrm{p}$ value $<0.05$

t-test for parametric data; Mann-Whitney U test for non-parametric data

All variables above presented in mean \pm SD except duration of dialysis presented in median (range) due to non parametric distribution.

Table (3): Optimization and bypass measures for post transplantation LUTD in both main groups

\begin{tabular}{|c|c|c|c|c|}
\hline \multicolumn{2}{|r|}{ Variable } & $\begin{array}{c}\text { Refluxing } \\
\text { kidney graft }\end{array}$ & $\begin{array}{l}\text { Non-refluxing } \\
\text { kidney graft }\end{array}$ & $\begin{array}{c}\mathbf{p} \\
\text { value }\end{array}$ \\
\hline \multirow{2}{*}{$\begin{array}{c}\text { Post Tx cause } \\
\text { specific surgery }\end{array}$} & No & $58(87.9 \%)$ & $79(100.0 \%)$ & \multirow{2}{*}{$0.001 *$} \\
\hline & Valve remnant ablation & $8(12.1 \%)$ & $0(0.0 \%)$ & \\
\hline \multirow{6}{*}{$\begin{array}{l}\text { Post TXs Bladder } \\
\text { Dysfunction } \\
\text { treatment }\end{array}$} & Anticholinergics & $8(21.1 \%)$ & $4(12.5 \%)$ & \multirow{6}{*}{0.193} \\
\hline & $\mathrm{CIC}$ & $8(21.1 \%)$ & $4(12.5 \%)$ & \\
\hline & Anticholinergics+ CIC & $12(31.6 \%)$ & $8(25.0 \%)$ & \\
\hline & Augmentation cystoplasty + CIC & $2(5.3 \%)$ & $4(12.5 \%)$ & \\
\hline & CIC with overnight indwelling catheter & $2(5.3 \%)$ & $0(0.0 \%)$ & \\
\hline & $\begin{array}{l}\text { Pre TXS defunctionalized bladder } \\
\text { improved after renal grafting }\end{array}$ & $6(15.8 \%)$ & $12(37.5 \%)$ & \\
\hline
\end{tabular}

* Significant difference at $p$ value $<0.05$; Chi Square test

Tx; Transplantation; CIC: clean intermittent catheterization

Baseline Characteristics with respect to Pre transplantation native kidney VUR and LUT condition.

64(44.1\%) patients with pre Tx VUR into native kidney; 41(62.1\%) patients in group A, while $23(29.1 \%$ ) patients in group $\mathrm{B}, \mathrm{P}$ value $=0.001$, and subdivided as following; In group A, Primary and secondary VUR occurred in
$13(31.7 \%)$, and $28(68.3 \%)$ patients, respecttively, while in group B primary and secondary reflux occurred in 10(43.5\%), and 13(56.5\%), respectively with no significance, $P$ value $=0.346 .38(57.6 \%)$ in group $\mathrm{A}$ and $31(39.2 \%)$ patients in group B had pre Tx abnormal LUT, $\mathrm{P}$ value $=0.028($ Table 4$)$ 
Table (4): pre Tx VUR and LUT condition in both main groups

\begin{tabular}{|c|c|c|c|}
\hline Variable & $\begin{array}{c}\text { Group (A) } \\
N=66\end{array}$ & $\begin{array}{c}\text { Group (B) } \\
\mathrm{N}=79\end{array}$ & P value \\
\hline \multicolumn{4}{|l|}{ Pre TXs native reflux with LUT condition } \\
\hline pre TXs VUR with normal LUT & $8(12.1 \%)$ & $9(11.4 \%)$ & \multirow{4}{*}{$<0.001^{*}$} \\
\hline pre TXs VUR with abnormal LUT & $33(50.0 \%)$ & $14(17.7 \%)$ & \\
\hline pre TXs NO VUR with normal LUT & $20(30.3 \%)$ & $39(49.4 \%)$ & \\
\hline pre TXs NO VUR with abnormal LUT & $5(7.6 \%)$ & $17(21.5 \%)$ & \\
\hline
\end{tabular}

*Statistically significant difference (Chi-square test).

LUT: lower urinary tract

Post transplant complications in groups (A) versus group (B):

No early graft failure or early death within the first year in all sample. No significant difference between groups regarding acute rejection episodes, early urological complications, lymphatic complications and infection complications except early vascular complications that was significant (table $5_{\mathrm{a}, \mathrm{b}}$ ). Regards to late complications, hypertension was the only significant complication in group A than B but other complications were not significant (Table 6). UTIs in group A occurred in 40(60.6\%) patients, while in group B occurred in $49(62 \%)$ patients, $\mathrm{P}$ value $=0.861,[\mathrm{OR}(95 \% \mathrm{CI})$ was $0.942(0.482-1.843)]$, but UTIs frequency (more than 3 episode per year) was happened in 20
(30.3\%) patients in group A, while 1(1.3\%) patient, $\mathrm{P}$ value $=0.001$. Febrile UTIs occurred in both groups [36 $(54.5 \%)$ patients in group A vs $39(49.4 \%)$ patients in group $\mathrm{B}$ ], $\mathrm{p}$ value $=$ 0.534 , but the frequency of febrile UTI(more than 3 episodes per year) occurred with significance [4 cases in group (A) versus no case in group (B)], $\mathrm{P}$ value $=0.026$. UTIs occurrence in group A subgroup as following; 14(43.8\%), 26(76.5\%) patients in group A1 and A2 respectively, $\mathrm{p}$ value $=0.008$, and the frequency of episodes (more than 3 per year) was also more in group A2 than A1 (11(32.4\%) vs $2(6.2 \%)$ patients, $\mathrm{p}$ value $=.001$. Regarding last culture proven UTIs, 40(60.6) patients in group A vs 42(53.2\%) patients in group B had culture proven UTI, $\mathrm{P}$ value $=0.368$.

Table ( $\left.5_{a}\right)$ : Showing early post operative complications

\begin{tabular}{|c|c|c|c|}
\hline Variable & $\begin{array}{c}\text { Group A } \\
\mathrm{N}=66\end{array}$ & $\begin{array}{c}\text { Group B } \\
\mathrm{N}=79\end{array}$ & p value \\
\hline \multicolumn{4}{|l|}{ Acute rejection } \\
\hline $\begin{array}{l}\text { No acute rejection } \\
\text { Acute rejection once } \\
\text { Acute rejection twice }\end{array}$ & $\begin{array}{c}38(57.6 \%) \\
25(37.9 \%) \\
3(4.5 \%) \\
\end{array}$ & $\begin{array}{l}36(45.6 \%) \\
33(41.8 \%) \\
1012.7 \%) \\
\end{array}$ & 0.150 \\
\hline \multicolumn{4}{|l|}{ Early graft failure } \\
\hline No & $66(100.0 \%)$ & $79(100.0 \%)$ & $\ldots$ \\
\hline \multicolumn{4}{|l|}{ Early urological complications } \\
\hline $\begin{array}{l}\text { No } \\
\text { Leakage managed by drainge PCN } \\
\text { Leakage needs exploration } \\
\text { Leakage managed by ureteral stenting } \\
\text { Failed ureteral stent removal }\end{array}$ & $\begin{array}{c}57(86.4 \%) \\
2(3.0 \%) \\
3(4.5 \%) \\
2(3.0 \%) \\
2(3.0 \%) \\
\end{array}$ & $\begin{array}{l}74(93.7 \%) \\
1(1.3 \%) \\
2(2.5 \%) \\
2(2.5 \%) \\
00.0 \%) \\
\end{array}$ & 0.505 \\
\hline \multicolumn{4}{|l|}{ Lymphatic complication } \\
\hline $\begin{array}{l}\text { No } \\
\text { Lymphocele managed conservatively } \\
\text { Lymphocele needed drainage } \\
\text { Lymphocele needed scelrosing } \\
\text { injection therapy }\end{array}$ & $\begin{array}{c}40(60.6 \%) \\
19(28.8 \%) \\
0(0.0 \%) \\
710.6 \%)\end{array}$ & $\begin{array}{c}5468.4 \%) \\
18(22.8 \%) \\
0(0.0 \%) \\
7(8.9 \%)\end{array}$ & 0.621 \\
\hline
\end{tabular}


Table ( $\left.5_{b}\right)$ : Showing early post operative complications

\begin{tabular}{|l|c|c|c|}
\hline \multicolumn{1}{|c|}{ Variable } & Group A & Group B & P value \\
\hline Infection complication & & & \\
\hline No & $53(80.3 \%)$ & $52(65.8 \%)$ & \\
Acute pyelonephritis & $0(0.0 \%)$ & $2(2.5 \%)$ & \\
EBV & $2(3.0 \%)$ & $4(5.1 \%)$ & \\
CMV & $10(15.2 \%)$ & $14(17.7 \%)$ & 0.235 \\
BK & $0(0.0 \%)$ & $1(1.3 \%)$ & \\
Sepsis & $1(1.5 \%)$ & $6(7.6 \%)$ & \\
\hline Early Vascular complications & & \\
\hline Perinephric hematoma that was managed conservatively & $8(12.1 \%)$ & $2(2.5 \%)$ & $0.043^{*}$ \\
\hline
\end{tabular}

All variables expressed in frequency and proportion

* Significant difference at $p$ value $<0.05$. Chi square test

EBV: Epstein Barr virus, CMV: Cytomegalovirus, BK: BK Polyomavirus

Table (6): Showing groups' late post operative complications

\begin{tabular}{|c|c|c|c|}
\hline Variable & $\begin{array}{c}\text { Group A } \\
(\mathrm{N}=66)\end{array}$ & $\begin{array}{l}\text { Group B } \\
(\mathrm{N}=79)\end{array}$ & $P$ value \\
\hline \multicolumn{4}{|l|}{ Hypertension } \\
\hline Yes & $31(47 \%)$ & $22(27.8 \%)$ & $0.017 *$ \\
\hline \multicolumn{4}{|l|}{ Hypertension therapy } \\
\hline $\begin{array}{l}\text { Monotherapy } \\
\text { Dual therapy } \\
\text { Triple therapy }\end{array}$ & $\begin{array}{c}20(64.5 \%) \\
8(25.8 \%) \\
3(9.7 \%) \\
\end{array}$ & $\begin{array}{c}9(40.9 \%) \\
10(45.5 \%) \\
3(13.6 \%) \\
\end{array}$ & 0.225 \\
\hline \multicolumn{4}{|l|}{ Late vascular } \\
\hline Renal artery stenosis & $1(1.5 \%)$ & $5(6.3 \%)$ & 0.220 \\
\hline \multicolumn{4}{|l|}{ Late urological complications } \\
\hline $\begin{array}{l}\text { Graft hydronephrosis due to obstruction } \\
\text { Graft hydronephrosis due to LUTD }\end{array}$ & $\begin{array}{l}0(0.0 \%) \\
4(6.1 \%)\end{array}$ & $\begin{array}{l}4(5.1 \%) \\
0(0.0 \%)\end{array}$ & $\begin{array}{c}0.064 \\
0.026^{*}\end{array}$ \\
\hline \multicolumn{4}{|l|}{ Late Death } \\
\hline Yes there was death after first year post Tx & $1(1.5 \%)$ & $2(2.5 \%)$ & $>0.99$ \\
\hline \multicolumn{4}{|l|}{ Chronic graft dysfunction } \\
\hline Yes & $37(56.1 \%)$ & $36(45.6 \%)$ & 0.208 \\
\hline \multicolumn{4}{|l|}{ Chronic rejection } \\
\hline Yes, there was chronic rejection & $5(7.6 \%)$ & $12(15.2 \%)$ & \multirow[t]{2}{*}{0.156} \\
\hline No & 61 & $67(84.8 \%)$ & \\
\hline
\end{tabular}

All variables expressed in frequency and proportion.

Significant difference at $p$ value $<0.05$. Chi square test

Post transplant radiological and FVC results in main groups:

In both U/S and VCUG, there was PVR in $66(100 \%)$ patients in group A and $14(17.7 \%)$ patients in group $\mathrm{B}, \mathrm{P}$ value $=0.001$, other results of VCUG were presented in table (7).
Graft reflux occurred in 60(90.9\%) cases and only $6(9.09 \%)$ cases had reflux into both the graft and the native kidney. Grades (G) 2, 3, 4, 5 VUR into renal graft occurred in 23, 34, 3, 6 patients respectively, while native kidney reflux which occurred in 6 cases had G2, and G3 in 4 
and 2 patients, respectively. UDs was done in 106 patients of all total sample $(65(98.4 \%)$ patients in group A, while $41(51.8 \%)$ patients in group B with all patients were awake in group A, but 6 patients only on sedation in group $\mathrm{B}, \mathrm{P}$ value $=0.001$. Mean MCC \pm SD was 421.2 $\pm 155.7(\mathrm{ml})$ in group A, while $408.6 \pm 144.4(\mathrm{ml})$ in group B, P value $=0.692$. We had VUR occurred with filling at low pressure in 32 cases, VUR occurred with filling at high pressure in 12 cases, VUR occurred with voiding at low pressure in 8 cases, VUR occurred with voiding at high pressure in 2 cases and lastly VUR occurred with both filling and voiding at high pressures in 12 cases.

FVC was done in 61(92.4\%) patients in group $\mathrm{A}$ and $68(86.1 \%)$ patients, $\mathrm{P}$ value $=0.224$, Nocturnal polyuria (NP) occurred in 43(66.2\%) patients in group $\mathrm{A}$ and $36(52.9 \%)$ patients group $\mathrm{B}, \mathrm{p}$ value $=0.121$. Urge incontinence and both NE plus urge incontinence occurred in 2 , and 11 patients, respectively in group $\mathrm{A}$, while occurred in 3 , and 7 patients, respectively in group A with no significance except NE that occurred in $12(18.2 \%)$ patients in group $\mathrm{A}$ and $4(5.1 \%)$ patients in group $\mathrm{B}, \mathrm{P}$ value $=0.012$.

Table (7): VCUG results in both main groups

\begin{tabular}{|c|c|c|c|c|c|}
\hline & \multicolumn{2}{|c|}{ Group (A) } & \multicolumn{2}{|c|}{ Group (B) } & \multirow[t]{2}{*}{$\mathrm{P}$ value } \\
\hline & \multicolumn{2}{|c|}{$\mathrm{N}=66$} & \multicolumn{2}{|r|}{$\mathrm{N}=79$} & \\
\hline \multicolumn{6}{|c|}{ Post transplantation VCUG bladder capacity } \\
\hline Normal bladder capacity & 1 & $(1.5 \%)$ & 64 & $(81.0 \%)$ & \multirow{3}{*}{$<0.001^{*}$} \\
\hline Decreased bladder capacity & 15 & $(22.7 \%)$ & 11 & $(13.9 \%)$ & \\
\hline Increased bladder capacity & 50 & $(75.8 \%)$ & 4 & $(5.1 \%)$ & \\
\hline Post TXs VCUG bladder capacity (ml) & \multirow{2}{*}{\multicolumn{2}{|c|}{$438.3 \pm 157.9$}} & \multirow{2}{*}{\multicolumn{2}{|c|}{$459.1 \pm 110.4$}} & \multirow{2}{*}{0.354} \\
\hline Mean \pm SD & & & & & \\
\hline \multicolumn{6}{|l|}{ Post TXs VCUG bladder wall } \\
\hline Normal wall & 43 & $(65.2 \%)$ & 68 & $(86.1 \%)$ & $0.003 *$ \\
\hline Irregular wall with serrations & 13 & $(19.7 \%)$ & 11 & $(13.9 \%)$ & 0.352 \\
\hline Irregular wall with diverticula & 6 & $(9.1 \%)$ & 0 & $(0.0 \%)$ & $0.006^{*}$ \\
\hline Hour glass deformity & 4 & $(6.1 \%)$ & 0 & $(0.0 \%)$ & $0.026^{*}$ \\
\hline \multicolumn{6}{|c|}{ Post transplantation VCUG posterior urethra } \\
\hline Dilated or funnel shape & 10 & $(15.2 \%)$ & 4 & $(5.1 \%)$ & $0.041^{*}$ \\
\hline \multicolumn{6}{|l|}{ Significant or Non significant PVR } \\
\hline Significant PVR & 20 & $(30.3 \%)$ & 5 & $(35.7 \%)$ & \multirow[t]{2}{*}{0.755} \\
\hline Non-significant PVR & 46 & $(69.7 \%)$ & 9 & $(64.3 \%)$ & \\
\hline
\end{tabular}

*Statistically significant difference.

PVR: post void residual. VCUG: voiding cystourethrograghy, 
Post transplant graft outcome, survival and patient survival in main groups

Asymptomatic VUR occurred in 30 cases (18 patients in group A1 and 12 patients in group A2), while symptomatic VUR occurred in 36 cases (14 cases in group A1 and 22 cases in group $\mathrm{A} 2, \mathrm{p}$ value $=0.087$ and managed as following: $(n=66)$, conservative by suppressive antibiotics only in 22 cases, anticholinergics + suppressive antibiotics only in 8 cases, anticholinergics + (clean intermittent catheterization) CIC + suppressive antibiotics in 12 cases, CIC+ suppressive antibiotics only in 10 cases, Redo ureteroneoystostomy in 10 cases, endoscopic Dextranomer/Hyaluronic acid injection in 2 cases and lastly augmentation cystoplasty in 2 cases. At follow up VCUG post reflux management showed resolution by grade in 63 cases and no resolution occurred in 3 cases ( 2 cases after endoscopic injection, 1 case after open redo repair). UTIs episodes per year in group A after VUR management as following no episodes occurred in 27 patients, one episode occurred in 31patients, 2 episodes occurred in 6 patients, 3 episodes occurred in 1 patient, more than 3 episodes in 1 patient.

No significant difference at creatinine level at different levels except creatinine level at time of discharge and at 3 months which was significant. Mean GFR \pm SD was $80.1 \pm 29.2(\mathrm{ml} / \mathrm{min})$ in group A and $86.5 \pm 30.7(\mathrm{ml} / \mathrm{min})$ in group B, $P$ value $=0.205$, also we didn't find any significance in GFR ( $\mathrm{ml} / \mathrm{min})$ between group (A1) vs group (A2) (Mean GFR $\pm \mathrm{SD}$ was
$81.3 \pm 30.5(\mathrm{ml} / \mathrm{min})$ in group (A1) vs $79 \pm 28.3$ $(\mathrm{ml} / \mathrm{min})$ in group $(\mathrm{A} 2), \mathrm{P}$ value $=0.76$. Median serum creatinine before VUR management was $1.2 \mathrm{mg} / \mathrm{dL}$ (range, 0.4-5.5), while after VUR management was $1 \mathrm{mg} / \mathrm{dL}$ (range, 0.4-5.5), P value $=0.001$.

$3(4.5 \%)$ patients in group A on dialysis, while $6(7.6 \%)$ patients in group $\mathrm{B}, \mathrm{p}$ value $=0.510$. There was one case death $(1.5 \%)$ in the group $\mathrm{A}$ but 2 cases $(2.5 \%)$ in group (B), P value $=0.99$. Graft nephrectomy was done due to dysfunctioning chronic rejected infected graft in one case $(1.5 \%)$ in group A, while was done in 2 cases $(2.5 \%)$ in group B, P value $=0.99$.

Kaplan-Meier analysis revealed no significant difference between main group in terms of mean graft survival time \pm SEM $[9.62 \pm 0.21 \mathrm{y}$, $(95 \% \mathrm{CI}, 9.2-10.04)]$ vs $[10.4 \pm 0.23 \mathrm{y},(95 \% \mathrm{CI}$, 9.96-10.85)], respectively and both 5 year survival rate and 10-year survival rate were $(100 \%$ vs $98.5 \%)$, and $(76.2 \%$ vs $84.7 \%)$ in group $\mathrm{A}$ and $\mathrm{B}$ respectively, $\mathrm{P}$ value $=0.942$ (Figure1). It also applied as life table for patient survival and there was no significant difference between both groups in terms of mean patient survival time \pm SEM $[10.76 \pm 0.23 \mathrm{y},(95 \% \mathrm{CI}$, $10.31-11.22)]$ vs [10.80 $\pm 0.14 \mathrm{y},(95 \% \mathrm{CI}, 10.54-$ 11.07)], respectively; $\mathrm{P}$ value $=0.977$ (Figure 2).

Linear regression analysis was done to the study the effects of multi independent variables as confounding factors on GFR (table 8).

Figure 1: Kaplan-Meier curves for post transplant graft survival over time using Log -rank statistics, significance was considered at $p$ value $<\mathbf{0 . 0 5}$
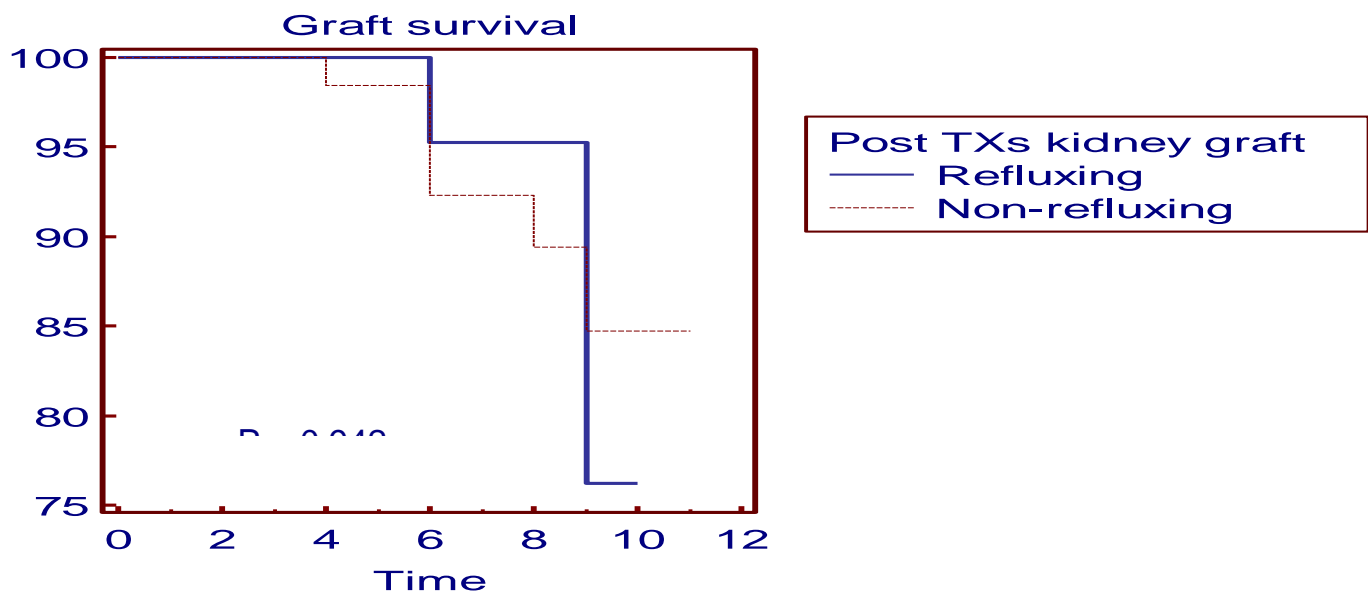
Figure 2: Kaplan-Meier curves for post transplant patient survival over time using Log rank statistics, significance was considerd at $\mathrm{p}$ value $<0.05$.

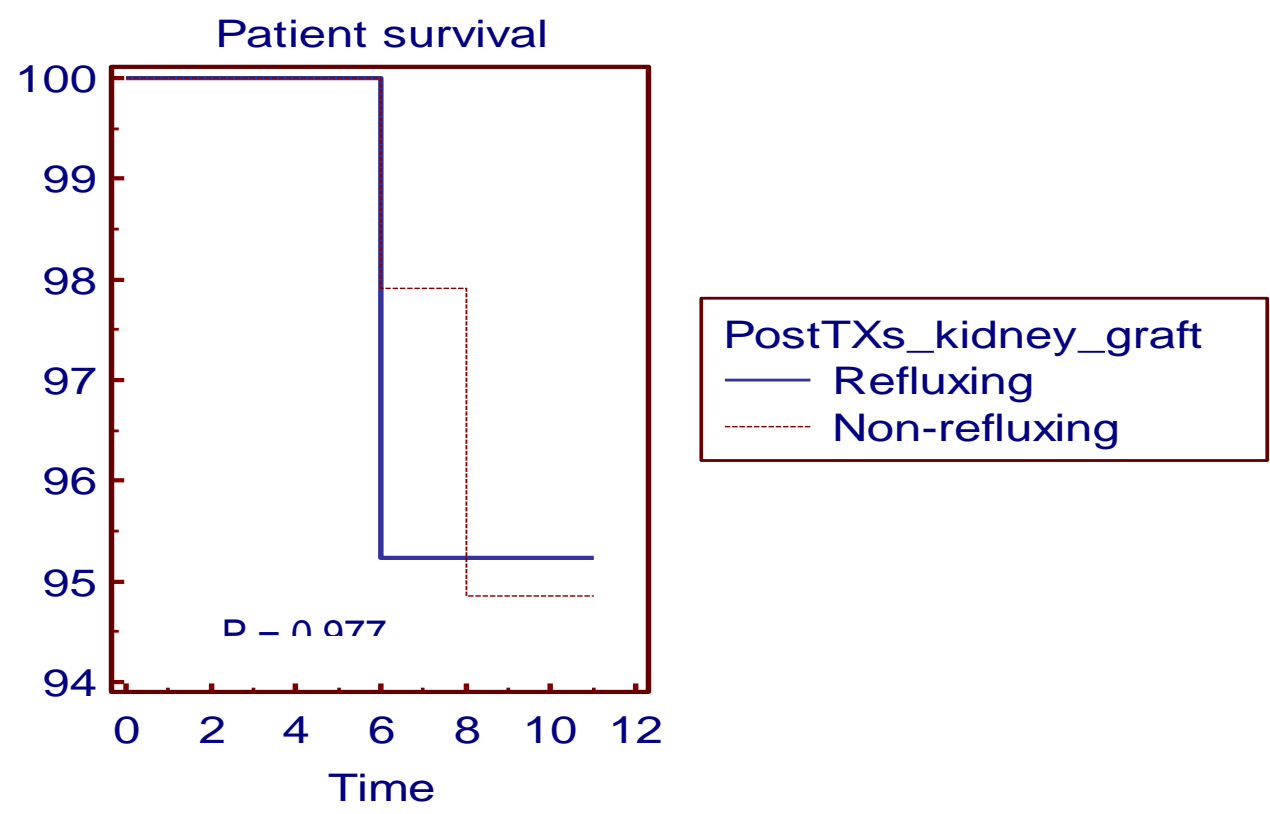

Table (8): Multivariate analysis of confounding independent factors to study all variables effects on estimated GFR using linear regression analysis model

\begin{tabular}{|c|c|c|}
\hline \multirow{2}{*}{ Variable } & \multicolumn{2}{|c|}{ Multivariate analysis } \\
\cline { 2 - 3 } & Standardized $\boldsymbol{\beta}$ & p value \\
\hline Chronic rejection proven biopsy & -0.356 & $<0.001^{*}$ \\
\hline UTI & -0.213 & $0.002^{*}$ \\
\hline Hypertension & -0.039 & 0.576 \\
\hline Acute rejection & -0.104 & 0.127 \\
\hline Last FK507 dose Toxicity & -0.261 & $<0.001^{*}$ \\
\hline Cortisone taking status & -0.189 & $0.004^{*}$ \\
\hline Time passed since transplantation & -0.109 & 0.106 \\
\hline
\end{tabular}

$\mathrm{R}^{2}=0.456$

*Statistically significant difference.

\section{Discussion}

This study monitored VUR effect in the context of Renal Tx patients and was conducted by a nearly uniform renal transplant team, and characterized by the stability of peri-operative measures done. The subjects were a relatively large retrospective cohort of children who received renal graft during or before the study. We had reflux in $45.5 \%$, while was $70 \%$ of total 31 patients in another study ${ }^{[2]}$ and reported to be $15 \%$ (1/15 patients after using modified barrytechnique ${ }^{[5]}$. Graft VUR is a well established risk factor for recurrent symptomatic UTIs. This form of graft VUR may lead to acute attacks of pyelonephritis due to recurrent ascending infections, so it should be appropriately diagnosed and treated espe-cially in the presence of recurrent UTI attacks ${ }^{[6]}$. This present study showed no differences between groups regarding age at time of transplant, donor criteria, and onset and duration of dialysis except pre-emptive Tx and 1/6 HLLA mismatch which were significant in group $\mathrm{B}$. The most common HLA cross-match type was 3/6HLA mismatch in both groups.

Regarding recipient demographics in our study, mean \pm SD age at time of Tx in group A and B was $8.7 \pm 3.4 \mathrm{y}$ and $8.9 \pm 2.8 \mathrm{yr}$ espectively, while the median (range) age was $9 \mathrm{y}$ (range $3-16$ ) in 
another study ${ }^{[5]}$ and was $20 \pm 6 y$ (mean \pm SD y), in Kara, E., et al., study ${ }^{[7]}$. 41 and 23 patients in group $\mathrm{A}$ and $\mathrm{B}$, respectively had reflux nephropathy as a primary pre Txcause, while $50 \%$ of total 62 cases due to reflux related nephropathy in Kara, E., et al., study ${ }^{[7]}$. Regarding pre Tx LUTD, we had 38 cases in refluxing graft group with LUTD in comparison to study done by Wang, Mary K., et al., ${ }^{[8]}$ there was 12 cases of total 37 cases. We had median follow up time (range) was 5(1-11)y, while in another study, median (range) follow up time was 23.7 months (range 6.3-39.3) ${ }^{[5]}$.

Postoperative urological complications such as ureteral stricture necrosis, and lastly urinary leakage may occur even after stenting ${ }^{[9]}$. In this study we had total 13 cases with post Tx urine leakage (7 in group A and 6 in group B) and 4 cases with ureteral stricture ( 2 in group $A$ and 2 in group B), while in the earlier original Barry puplication, there was high rate of urological complications which decreased after using modified barry technique in which short tunnel length about $2 \mathrm{~cm}$ was used so less distal ureter ischemia and less stricture ${ }^{[5]}$, this difference can be explained by the length of distal ureter which underneath the tunnel.

It is our institution protocol to use antireflux ureteral anastomosis by Lich-Gregoir technique and stenting the ureter in all cases at time of Tx. Also, we did Lich-Gregoir Redo ureteroneocystotomy in $10(15.5 \%)$ cases of total 66 cases, while in a study was done by Turunç, V., et al., ${ }^{[10]}$ who used extra vesicalsero muscular tunnel lengthening technique with at least $3 \mathrm{~cm}$ tunnel length in 20(52.6\%) cases of 38 cases and there was no difference regarding follow up VCUG, (18(90\%) patients resolution by grade in that study, the same as our study results, 9 of 10 cases $(90 \%)$ resolution in our study. In the previous study, they did anastomosis to native ureter (uteterouretrostomy) in 18 patients with a percentage $47.4 \%$, that we didn't use it at all as most of native kidneys in our pediatrics were nephroctomized and it isn't in our institution protocol to use the native ureter in anastomosis.

As known that repair of primary VUR decreases the frequency of febrile UTI episodes ${ }^{[1]}$. Likewise, in our present study, we found that febrile UTIs attacks decreased dramatically after VUR correction (no episodes in 27 cases).
As described by Turunç, V., et al., ${ }^{[10]}$, median serum creatinine before VUR correction was $1.4 \mathrm{mg} / \mathrm{dL}$ (range, 0.8-4.3) and was $1.2 \mathrm{mg} / \mathrm{dL}$ (range, 0.7-4.5) after peration with no significant difference, but in this present study, median serum creatinine before VUR correction was $1.2 \mathrm{mg} / \mathrm{dL}$ (range, 0.4-5.5), while after VUR management was $1 \mathrm{mg} / \mathrm{dL}$ (range, 0.45.5), $\mathrm{P}$ value $=0.001$, this significance $\mathrm{can}$ be explained by that we didn't correct only reflux but we did optimization and bypass measures for LUT defunctionalization but in their puplication they did correction for reflux only in optimal LUT condition ${ }^{[10]}$.

Regarding to endoscopic VUR correction we didn't use it in large cohort group due to low success rate and we did endoscopic Dextranomer/Hyalouronic acid injection in only 2 cases for symptomatic low grade reflux but no resolution and failure was the end as same as the results obtained by Latchamsetty ${ }^{[12]}$ et al., who used collagen injection in 7 patients and all patients had persistent VUR. In both studies, failure attributed to anatomical difference between native orifice, which lie on a wellsupported trigonal area opposite to transplant neo-orifices which wasn't well supported and this was a possible explanation for the failure. Regarding reflux grades, we had G2,G3,G4, and $\mathrm{G} 5$ in 23, 34, 3, and 6 patients, respectively, while in the study done by Wang, Mary K., et al., ${ }^{[7]}$, there was G3, and (G4-5)collectively in 15 , and 18 patients, respectively with more advanced grades in their study than our study.

Regarding group A2 and A1 subgroups, the eGFR was $79 \pm 28.3(\mathrm{ml} / \mathrm{min})$ and $81.3 \pm 30.5(\mathrm{ml}$ /min) in group A2 and group A1, respectively with no significance, in line with the results obtained by Wang, Mary K., et al., ${ }^{[7]}$ in which there was 25 patients with LUT abnormality and 12 patients with normal LUT of total 37 cases and there was no significant difference in GFR levels regarding LUT condition [Median (range) 76 (63-88), in abnormal LUT group vs 77(67-107), in normal LUT group with no significance also, One possible explanation due to optimization of LUT function either pre Tx or post Tx with optimal bypass measures and the important issue is optimum patient selection and good post operative follow up.

Renal Graft Vesicoureteral Reflux Survival and Oucome in Pediatric Recipients; Single Centre Experience 
This study limitations included absence of random allocation due to nature of the study and normal control which is difficult to obtain due to ethical and methodology obstacles, also due to absence of more or less equal starting points of the cases in both study groups, also at each group; cases were included with different diseases and different management interval. Another limitation, we don't have video UDS to know at which volume the reflux will occur and to measure MCC and reflux PVR accurately .From financial point also, we used Shwartz formula to evaluate GFR as we don't have the financial facilities to do renal isotopic scan. Lastly a part of this study was retrospective designed so I hope to increase more cohort cases in future.

\section{Conclusions}

Graft VUR in the setting of pediatric $\mathrm{Tx}$ is very common and not always require surgical repair and asymptomatic graft VUR needs just follow up. The goals of correcting graft symptomatic reflux is identical to those of treating symptomatic primary VUR, which are to prevent further renal infections, scarring and hypertension. No difference between refluxing and non refluxing graft in terms of graft survival and outcome. Post transplantation VCUG shouldn't be used as a routine protocol to diagnose VUR, as it is asymptomatic in most cases and we recommend doing it only in selected cases when indicated like patients with recurrent UTIs as the possibility of having reflux in these scenarios is high.

\section{References}

1. Margreiter, M., Györi, G.P., Böhmig, G.A., Trubel, S., Mühlbacher, F. and Steininger, R., 2013. Value of routine voiding cystourethrography after renal transplantation. American Journal of Transplantation, 13(1), pp.130-135.

2. Coulthard, M.G. and Keir, M.J., 2006. Reflux nephropathy in kidney transplants, demonstrated by dimercaptosuccinic acid scanning.Transplantation,82(2),pp.205-210

3. Kayler, L., Kang, D., Molmenti, E. and Howard, R., 2010, June. Kidney transplant ureteroneocystostomy techniques and complications: review of the literature. In Transplantation proceedings (Vol. 42, No. 5, pp. 1413-1420).
4. Akiki, A., Boissier, R., Delaporte, V., Maurin, C., Gaillet, S., Karsenty, G., Coulange, C. and Lechevallier, E., 2015. Endoscopic treatment of symptomatic vesicoureteral reflux after renal transplantation. The Journal of urology,193(1),pp.225-229.

5. Vasdev, N., Coulthard, M.G., Lambert, H., Keir, M., Wyrley-Birch, H., Rix, D., Soomro, N. and Talbot, D., 2012. The modified Barry technique to prevent vesicoureteric reflux in paediatric renal transplant recipients: Initial recipient outcomes. Journal of Pediatric Urology, 8(1), pp.97-102.

6. Barrero, R., Fijo, J., Fernandez-Hurtado, M., García-Merino, F., León, E. and Torrubia, F., 2007. Vesicoureteral reflux after kidney transplantation in children. Pediatric transplantation,11(5),pp.498-503.

7. Kara, E., Sakaci, T., Ahbap, E., Sahutoglu, T., Koc, Y., Basturk, T., Sevinc, M., Akgol, C., Kayalar, A.O., Ucar, Z.A. and Unsal, A., 2016, July. Posttransplant urinary tract infection rates and graft outcome in kidney transplantation for endstage renal disease due to reflux nephropathy versus chronic glomerulonephritis. In Transplantation proceedings (Vol. 48, No. 6, pp. 2065-2071).

8. Wang, M.K., Chuang, K.W., Li, Y., Gaither, T., Brakeman, P., Gonzalez, L., Brennan, J. and Baskin, L.S., 2018. Renal function outcomes in pediatric patients with symptomatic reflux into the transplanted kidney treated with redo ureteroneocystostomy. Journal of pediatric urology, 14(3), pp.275-e1

9. Pichler, R., Buttazzoni, A., Rehder, P., Bartsch, G., Steiner, H. and Oswald, J., 2011. Endoscopic application of dextranomer/hyaluronic acid copolymer in the treatment of vesico-ureteric reflux after renal transplantation. BJU international, 107(12), pp.1967-1972.

10. Turunç, V., Eroğlu, A., Tabandeh, B. and Erol, A., 2017, April. Comparison of Surgical Correction Techniques for PostRenal Transplantation Vesicoureteral Reflux. In Transplantation Proceedings (Vol. 49, No. 3, pp. 512-516).

11. Ranchin, B., Chapuis, F., Dawhara, M., Canterino, I., Hadj-Aïssa, A., Saïd, M.H., Parchoux, B., Dubourg, L., Pouillaude, J.M., Floret, D. and Martin, X., 2000. 
Vesicoureteral reflux after kidney transplantation in children. Nephrology Dialysis Transplantation, 15(11), pp.1852-1858.

12. Latchamsetty, K.C., Mital, D., Jensik, S. and Coogan, C.L., 2003, June. Use of collagen injections for vesicoureteral reflux in transplanted kidneys. In Transplantation proceedings (Vol. 35, No. 4, pp. 1378-1380). 